\title{
Hypersensitivity Reactions to Contrast Media: Prevalence, Risk Factors and the Role of Skin Tests in Diagnosis - A Cross-Sectional Survey
}

\author{
Ozlem Goksel $^{\mathrm{a}}$ Omur Aydın ${ }^{\mathrm{a}}$ Cetin Atasoy $^{\mathrm{b}}$ Serdar Akyar ${ }^{\mathrm{b}}$ \\ Yavuz Selim Demirel $^{\text {a }} \quad$ Zeynep Misirligil ${ }^{\text {a }}$ Sevim Bavbek ${ }^{a}$ \\ a Department of Pulmonary Diseases, Division of Allergy and Clinical Immunology, and bepartment of Radiology, \\ School of Medicine, Ankara University, Ankara, Turkey
}

\section{Key Words}

Drug allergy $\cdot$ Drug hypersensitivity $\cdot$ Contrast media $\cdot$

Skin tests $\cdot$ Computerized tomography

\begin{abstract}
Background: Hypersensitivity to contrast media (CMs) may be common and serious. Aim: To evaluate the prevalence of CM hypersensitivity, risk factors associated with it and the role of skin testing in its diagnosis. Methods: A structured questionnaire was administered to patients who underwent computed tomography during a 1-year period. Skin tests with CMs, including skin prick tests (SPTs), intradermal tests (IDTs) and patch tests (PTs), were conducted on CM reactors $(n=24)$. Volunteers who tolerated CM exposure or had never been exposed to any CMs served as controls $(n=37)$. Results: A total of 1,131 patients ( 630 females and 501 males; mean age $55 \pm 14.2$ years) were enrolled in the study. The prevalence of historical and current CM reactors was 33/1,131 $(2.92 \%)$ and $8 / 1,105(0.72 \%)$, respectively. The skin was the most affected site, with mild to moderate reactions. Female gender, a history of doctor-diagnosed asthma, drug allergy, food allergy and psychiatric diseases were significant risk factors. The sensitivities of SPTs and early readings of IDTs in the diagnosis of immediate reactions were 0 and $20 \%$, respectively, and the specificities were 94.6 and $91.4 \%$, respectively. For early readings of IDTs, the positive predictive value
\end{abstract}

(PPV) and negative predictive value (NPV) were 40 and $80 \%$, respectively. For nonimmediate reactions, the sensitivities of delayed readings of IDTs and PTs were 14.3 and $25 \%$, respectively; specificity was $100 \%$ for both tests. The PPV was $100 \%$ for both of these tests, and the NPVs were 85.4 and $82.4 \%$, respectively. Conclusions: Our findings are comparable with the incidence, profile and risk factors associated with CM hypersensitivity reported previously. Skin testing with CMs has a high specificity, but its role in diagnosis is limited due to low sensitivity.

Copyright $\odot 2011$ S. Karger AG, Basel

\section{Introduction}

Iodinated contrast media (CMs) are among the most widely used drugs worldwide [1]. They were introduced into clinical practice in the 1950s, and since then about 75 million X-ray examinations have been performed yearly with the use of CMs. Moreover, the number of patients receiving these medications continues to rise with the increasing use of computed tomography (CT) [2]. Although they are chemically inert and regarded as relatively safe, hypersensitivity reactions to CMs ranging in severity from mild to severe, life-threatening reactions may still occur $[3,4]$.

\section{KARGER}

Fax +41613061234 E-Mail karger@karger.ch www.karger.com

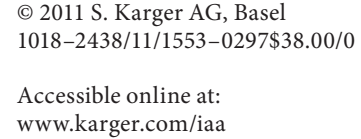

Correspondence to: Prof. Sevim Bavbek

Division of Pulmonary Disease, Department of Allergy, Cebeci Hospital

School of Medicine, Ankara University

TR-06530 Ankara (Turkey)

Tel. +90 312595 6581, Fax +90 312319 0046, E-Mail bavbek@ medicine.ankara.edu.tr 
Iodinated CMs are categorized into ionic and nonionic types. The prevalence rate of CM-induced hypersensitivity reactions was first evaluated through phase II and III clinical trials and then through large-scale postmarketing surveillance studies [4-6]. Since the introduction of nonionic CMs into clinical practice, adverse reactions associated with CM administration have been reported less frequently [5, 7-10]. A large-scale nationwide study in Japan reported that not only the prevalence of all degrees of adverse reactions to CMs but also the prevalence of severe and potentially life-threatening adverse reactions were significantly reduced with nonionic CMs [6].

Adverse reactions after CM administration have been divided into 3 types, namely allergic and nonallergic hypersensitivity reactions, toxic reactions and reactions unrelated to CM application [11]. Hypersensitivity reactions after exposure to CMs are classified according to the time interval between CM administration and the first appearance of clinical manifestations as immediate (reaction within $1 \mathrm{~h}$ ) and nonimmediate reactions (reaction after $1 \mathrm{~h}$ ). Although the exact mechanism underlying the pathogenesis of CM-induced allergy-like reactions is not completely understood, recent studies indicate that a subset of immediate reactions are likely true IgE-mediated reactions, while most of the nonimmediate skin reactions are most likely caused by $\mathrm{T}$ cells $[2,7,12,13]$. Thus, patients who experience such hypersensitivity reactions are advised to undergo allergologic evaluation, including skin tests. However, limited data are available about the value of skin tests in the diagnosis of CM-related hypersensitivity reactions $[1,2,12,14,15]$.

In Turkey, CMs have been used for radiological evaluation since the introduction of these drugs; however, there are no data documenting the frequency and type of hypersensitivity reactions to either ionic or nonionic CMs. Therefore, in this study we aimed to determine the prevalence of hypersensitivity reactions to CMs as well as the risk factors associated with them and to assess the role of skin testing in the diagnosis of CM-related hypersensitivity reactions.

\section{Materials and Methods}

\section{Patient Selection and Study Design}

The Departments of Allergy and Clinical Immunology and Radiology of the Ankara University School of Medicine jointly conducted this descriptive, prospective, open-label, 1-year study between June 2006 and June 2007. In the first part, subjects who presented to the Radiology Department for CT scan were consecutively enrolled to determine the prevalence and risk factors of hypersensitivity reactions to $\mathrm{CM}$. The patients were questioned with the help of a radiology nurse using a modified European Network of Drug Allergy questionnaire [16], including demographic characteristics, previous CM exposure, previous CM-related hypersensitivity reactions and comorbid diseases.

Clinical manifestations of immediate and nonimmediate hypersensitivity reactions to CMs were defined according to Brockow et al. [14]. The severity scale of Ring and Messmer [17] was used for classification of immediate reactions as grade 1 (generalized cutaneous and/or mucocutaneous symptoms), grade 2 (mild systemic reactions), grade 3 (life-threatening systemic reactions) or grade 4 (cardiac and/or respiratory arrest). Nonimmediate reactions were graded as mild when no treatment was required, moderate when the patient responded readily to appropriate treatment and no hospitalization was needed and severe when the reaction required hospitalization or was life-threatening $[8,14]$.

The second part of the study was dedicated to evaluating the role of skin testing in the diagnosis of hypersensitivity reactions to CMs. Patients with a reliable history of verbal or documented CM hypersensitivity were included in the study. They either had a history of hypersensitivity reactions to CMs before entering the study and needed a new radiologic evaluation with CMs or experienced a CM-induced reaction during the 1-year study period. In the first part of the study, two investigators, O. Göksel and O. Aydin, who are specialists in allergy, interviewed patients who had had previous reactions to CMs about the type and severity of the reactions by telephone. In the second part of the study, patients who developed a reaction during the CT examination were all referred to the Allergy Department from the Radiology Department. The same investigators evaluated these patients whether they had a hypersensitivity reaction to CMs or not.

Additionally, patients only with a history of CM-induced allergy-like reactions from the end of the first part of the study in June 2007 to June 2009 were included in order to increase the number of CM reactors. Volunteers who tolerated CM exposure or had never been exposed to any CM served as controls. The Ethics Committee of Ankara University approved the survey, and informed consent was obtained from the study participants.

\section{Skin Testing for CM-Induced Immediate and Nonimmediate}

Reactions

Skin tests, including skin prick tests (SPTs), intradermal tests (IDTs) and patch tests (PTs), with CMs were performed according to the type of reactions induced by CMs $[4,14,18]$. Positive (histamine, $0.01 \%$ ) and negative (saline solution) controls were also included in skin testing. An SPT with the culprit CM (full strength) was performed on the volar forearm and was read after $15 \mathrm{~min}$. A wheal reaction with a mean diameter $3 \mathrm{~mm}$ greater than that of the negative control was considered positive $[2,14]$. If it was negative, the first IDT was performed with 1:1,000-diluted CM on the volar forearm or upper arm. An amount of $0.03-0.05 \mathrm{ml}$ of the test solution was injected into the skin to produce a bleb of $3-5 \mathrm{~mm}$ in diameter. If the result was negative after $15 \mathrm{~min}$, subsequent IDTs with 100 - and 10 -fold diluted CM were performed. Readings were performed after 15 min (early reading) and on days 1, 2 and 3 (delayed readings). The IDT was considered as $1+$ positive if the size of the wheal was between 3 and $5 \mathrm{~mm}, 2+$ positive if the size of the wheal was between 6 and $10 \mathrm{~mm}$ and $3+$ positive if the size of the wheal was between 11 and $15 \mathrm{~mm}$ on the early readings or if an erythematous induration at the skin test site was present on the 
delayed readings $[14,19]$. For the PTs, since there were no standardized and commercially available CM allergens for PTs, 1,000-, 100and 10 -fold dilutions of the culprit CM in saline were prepared by researchers at the Department of Immunology and Allergy. Solutions were soaked in a plastic chamber on a filter paper (Haye's Test Chamber; Haye's Service B.V., The Netherlands) and fixed on the patient's back with a special nonirritant, nonsensitized adhesive tape strip (Haye's) for 3 days. European Standard Series PTs were also applied to exclude any nonimmediate reactions induced by another contact sensitizer allergen (Brial Allergen, Greven, Germany). Readings were conducted $15 \mathrm{~min}$ after removal of the strips and also after 24, 48 and $72 \mathrm{~h}$ in accordance with the recommendations of the European Society of Contact Dermatitis [18].

Subsequently, the role of SPTs and early readings of IDTs in the diagnosis of immediate reactions, and the role of PTs and delayed readings of IDTs in the diagnosis of nonimmediate reactions were statistically evaluated. The patients who were given premedication before radiological evaluation and the patients who were tested with an alternative CM because the culprit agent was unknown were not included in these statistical analyses. An observation or a reliable/documented medical history of an allergy-like reaction after administration of CM was used as the gold standard, and skin test sensitivity and specificity were calculated accordingly along with the controls.

\section{Evaluation of Atopy}

Patients skin tested with CM were also skin tested with inhalant allergens for the diagnosis of atopic status. Atopy was defined as a positive SPT reaction to at least one of the aeroallergens tested. Glycerinated extracts (Stallergenes, Antony, France) of the following allergenic sources were used in SPTs: Dermatophagoides pteronyssinus, Dermatophagoides farinae, cockroach, grass, tree, weed pollens, cat, dog and Alternaria and Cladosporium antigens. Positive (histamine, $10 \mathrm{mg} / \mathrm{ml}$ ) and negative (saline solution) controls were also included in the SPTs. The puncture method with a 1-mm-tip disposable lancet was used, and a wheal reaction with a mean diameter $3 \mathrm{~mm}$ greater than that of the negative control was considered a positive response.

\section{Statistical Methods}

Statistical analyses were performed using the Statistical Package for Social Sciences for Windows (SPSS version 11.0; SPSS, Chicago, Ill., USA). Descriptive statistics are expressed as means \pm SD and numbers with percentages. Categorical data were analyzed by $\chi^{2}$ test. Univariate analyses were performed with Fisher's exact test, while logistic analyses were used for multiple analyses. The odds ratio and its $95 \%$ confidence interval (CI) were calculated. A p value of $<0.05$ was considered statistically significant.

Sensitivity of SPTs or early readings of IDTs was defined as the proportion of subjects with a positive CM-induced immediate hypersensitivity reaction whose SPTs or IDTs were positive with the culprit CM. Specificity was defined as the proportion of control group subjects whose SPTs or IDTs were negative with the culprit CM. The same method was used for the determination of the sensitivity and specificity of delayed readings of IDTs and PTs. The positive predictive value (PPV) was calculated as follows: number of true positives/(number of true positives + number of false positives). The negative predictive value (NPV) was calculated as follows: number of true negatives/(number of true negatives + number of false negatives).

Hypersensitivity to Contrast Media

\section{Results}

\section{Prevalence and Risk Factors Associated with} CM-Induced Hypersensitivity Reactions

In the first part of the study, 1,131 subjects with a mean age of $55 \pm 14.2$ years filled out the European Network of Drug Allergy questionnaire during the 1-year period. Thirty-five of the 1,131 patients had a history of a CMinduced hypersensitivity reaction. Among them, 33 were considered to have a reliable history and 2 patients who had hypertension and reported increases in their blood pressure after CM administration were excluded from the study. For 12 of the 33 patients, their past reactions were later documented by a note written by a physician who had witnessed and/or treated the reaction. Therefore, the past prevalence of CM-induced hypersensitivity reactions was $2.92 \%(33 / 1,131$; table 1$)$. Among the 33 patients, 21 and 12 patients were defined as having had immediate- and non-immediate-type reactions, respectively. Seven (4 immediate-type, 3 non-immediate-type reactions) of the 33 had further CT evaluation with a CM that was negative on skin testing. Of these 7 , iohexol was the culprit CM in 2 patients, and the culprit CM could be determined as an ionic CM in another 2 patients; however, the culprit CM could not be identified in the other 3 patients. The Radiology Department conducted further CTs without using a CM for the remaining 26 cases. Thus, a CM from the nonionic iodinated monomer or dimer group was administered by the intravenous route in 1,097 patients and by the intraarterial route in 8 patients. The most frequently used CM was iohexol, which was given to 784 subjects $(69.3 \%)$, followed by iomeprol in 129 cases (11.4\%), iopromide in 79 cases (7\%), ioxilan in 73 cases (6.5\%), iopamidol in 23 cases (2\%), iodixanol in 17 cases (1.5\%) and iobitridol in 1 case $(0.1 \%)$.

In the study group, $69.5 \%$ of the patients had prior CM exposure and $64.9 \%$ had at least one comorbid disease. While the comorbid disease was one of the nonallergic diseases in $43.7 \%$ of the patients, $10.8 \%$ had at least one disease that could be of allergic origin. Asthma was the most frequently observed disease of potential allergic origin (4.4\%). A total of 41 patients (3.6\%) reported hypersensitivity to drugs, among which penicillin and analgesics were most frequent. There were only 5 patients with a history of food allergy; namely, 1 patient to cacao, 1 to mushroom, 1 to gluten and 2 to peach (table 1 ).

Eight patients (current prevalence: 8/1,105, 0.72\%) experienced a hypersensitivity reaction to a CM administered during the 1-year study period. Among them, 4 experienced immediate-type reactions (grade 1-2) and 4 
Table 1. Characteristics of the study group and prevalence of CMinduced hypersensitivity reactions

\begin{tabular}{lc}
\hline Characteristic & \\
\hline Total patients & 1,131 \\
\hline Males/females & $501 / 630(44.3 / 55.7)$ \\
\hline Mean age (range), years & $55 \pm 14.2(18-88)$ \\
\hline Prior exposure to CM & $786(69.5)$ \\
\hline Prior CM-induced reaction & $33(2.92)$ \\
\hline CM exposure for the first time & $345(30.5)$ \\
\hline Patients with comorbid diseases & $584(51.6)$ \\
\hline Patients with comorbid allergic diseases & $122(10.8)$ \\
Patients with 1 allergic disease & $104(9.2)$ \\
Patients with 2 allergic diseases & $12(1.1)$ \\
Patients with 3 allergic diseases & $6(0.5)$ \\
\hline
\end{tabular}

\section{Comorbid allergic diseases}

\begin{tabular}{lc} 
Asthma & $50(4.4)$ \\
History of drug allergies & $41(3.6)$ \\
$\quad$ Penicillin allergy & $21(1.9)$ \\
$\quad$ NSAID allergy & $8(0.7)$ \\
Allergic rhinitis & $17(1.5)$ \\
Chronic urticaria/angioedema & $11(1)$ \\
Contact dermatitis & $11(1)$ \\
Metal allergy & $10(0.9)$ \\
Food allergy & $5(0.4)$ \\
Venom allergy & $1(0.08)$ \\
\hline Patients with comorbid nonallergic diseases & $494(43.7)$ \\
Hypertension & $257(22.7)$ \\
Heart disease & $119(10.5)$ \\
Liver disease & $104(9.2)$ \\
Thyroid disease & $80(7.1)$ \\
Diabetes mellitus & $52(4.6)$ \\
Renal disease & $44(3.9)$ \\
Gastrointestinal system disease & $40(3.5)$ \\
Psychiatric disease & $24(2.1)$
\end{tabular}

\begin{tabular}{lr}
\hline Reaction to $C M$ in the past $(n=1,131)$ & \\
Immediate reaction & $21(1.86)$ \\
Nonimmediate reaction & $12(1.06)$ \\
\hline Reaction to CM during the study $(n=1,105)$ & \\
Immediate reaction & $4(0.36)$ \\
Nonimmediate reaction & $4(0.36)$ \\
\hline Total reactions $(n=1,131)$ & $41(3.62)$ \\
Immediate reactions & $25(2.21)$ \\
Nonimmediate reactions & $16(1.41)$
\end{tabular}

Values represent numbers of patients, with percentages in parentheses, except where indicated otherwise. NSAID = Nonsteroidal anti-inflammatory drug.
Table 2. Clinical manifestations of hypersensitivity reactions to CMs

\begin{tabular}{lll} 
Symptoms & $\begin{array}{l}\text { Immediate } \\
\text { reaction } \\
(\mathrm{n}=25)\end{array}$ & $\begin{array}{l}\text { Nonimm } \\
\text { reaction } \\
(\mathrm{n}=16)\end{array}$ \\
\hline $\begin{array}{l}\text { Itching, erythema and urticaria- } \\
\quad \text { like skin rashes }\end{array}$ & $11(44)$ & $3(18.7)$ \\
$\begin{array}{l}\text { Maculopapular exanthems } \\
\text { Angioedema }\end{array}$ & - & $6(37.5)$ \\
Dyspnea & $2(8)$ & $7(43.7)$ \\
Nausea/abdominal cramp & $9(36)$ & $4(25)$ \\
Vomiting/diarrhea & $8(32)$ & - \\
Skin exfoliation & $9(36)$ & - \\
Fixed drug eruption & - & $1(6.2)$ \\
Hypotension & - & $1(6.2)$ \\
Syncope & $6(24)$ & $4(25)$ \\
\hline
\end{tabular}

Values represent numbers of patients, with percentages in parentheses.

experienced non-immediate-type reactions (mild to moderate; table 1). Iohexol was the culprit drug in 7 of these patients, and 1 reacted to iopromide. The most frequently reported symptoms were maculopapular exanthems in $37.5 \%$, followed by itching, erythema and urticaria-like skin rashes in $18.7 \%$, angioedema in $21.9 \%$, dyspnea in $24.4 \%$, hypotension in $24.4 \%$, nausea and vomiting in $21.9 \%$, syncope in $7.3 \%$, bullous exanthema and exfoliative eruption in $2.4 \%$ and change of color at a fixed skin site in $2.4 \%$. A summary of the clinical presentations of past and current CM reactors $(n=41)$ is shown in table 2. No hospitalization or mortality was observed in these patients, and all reactions were well controlled with systemic steroids and antihistamines.

There were significant differences between the patients with past/current CM-induced hypersensitivity reactions $(\mathrm{n}=41)$ and patients who tolerated CMs without any reaction $(n=1,090)$ in terms of gender and a history of doctor-diagnosed allergic or nonallergic disease. In multivariate analysis, female gender, a history of doctordiagnosed asthma, drug allergy, food allergy or psychiatric diseases emerged as significant risk factors associated with CM-induced hypersensitivity reactions (table 3).

\section{Skin Test Results}

Skin tests were performed within 2 months to 27 years after the CM-induced hypersensitivity reaction. A total of 24 reactors to CMs (18 females and 6 males; mean age $51 \pm 14.90$ years) were skin tested with an alternative CM or culprit agent. Among them, there were 7 patients with 
Table 3. Risk groups with respect to CM-induced hypersensitivity reactions

\begin{tabular}{lcllc}
\hline Risk factor & CM reactors $(\mathrm{n}=41)$ & $\mathrm{p}$ univariate & p multivariate & Odds ratio (95\% CI) \\
\hline Female gender & $29(65.9 \%)$ & $<0.01$ & $<0.05$ & $2.17(1.037-3.924)$ \\
Psychiatric disease & $6(13.6 \%)$ & $<0.001$ & $<0.01$ & $6.372(2.123-19.131)$ \\
Drug allergy & $8(18.2 \%)$ & $<0.001$ & $<0.001$ & $5.738(2.293-14.357)$ \\
Asthma & $8(18.2 \%)$ & $<0.001$ & $<0.01$ & $4.345(1.769-10.672)$ \\
Food allergy & $3(6.8 \%)$ & $<0.01$ & $<0.05$ & $7.761(1.158-52.004)$ \\
\hline
\end{tabular}

a history of CM hypersensitivity prior to the study, 8 patients with reactions to CMs during the 1-year study period and 9 patients with reactions to CMs after the 1 -year study period until June 2009. Of the last 9 patients, 6 had immediate reactions ( 4 with grade 2, 2 with grade 1 ) and 3 had nonimmediate reactions ( 2 mild, 1 moderate). The 7 prior reactors (No. 1-7, table 4) were the first cases on whom skin testing was performed since they needed CT evaluation with a CM. In 2 patients, the culprit CM was documented as iohexol, while in another 2 the culprit agent was a generic, unknown ionic CM. The culprit agent could not be determined in the remaining 3. Of these 7 patients, iohexol was skin tested in 5 patients, including 2 iohexol reactors, and ioversol and ioxilan were each skin tested in 1 patient before radiological evaluation (table 4). SPTs and IDTs were all negative in these 7 patients. Nevertheless, as the sensitivity and specificity of the skin tests for CM hypersensitivity were not exactly known, these cases were offered a standard premedication protocol (40 mg of methylprednisolone 13,7 and $1 \mathrm{~h}$ before CT, $50 \mathrm{mg}$ of pheniramine $1 \mathrm{~h}$ before) for CT application, but only 6 of them were premedicated by the radiology department (No. 2-7, table 4). No reaction was observed with CMs in these premedicated patients. However, 1 prior reactor (patient No. 1, table 4) who had a history of a grade 1 immediate reaction to an unknown CM experienced a grade 2 immediate reaction when CT was performed with ioxilan without premedication despite a negative result on skin testing with ioxilan.

Skin tests were positive in 2 of the 8 patients who developed CM-related hypersensitivity reactions during the study period (No. 8-15, table 4) and in 3 of the additional 9 patients with reactions to CMs after the study period (No. 16-24, table 4).

Almost all 24 patients were skin tested with only 1 $\mathrm{CM}$, either culprit or alternative, and 1 patient was tested with 2 CMs. The culprit agent was known and tested in 19 patients but it could not be identified in 5 patients. Out of the 24 patients who were skin tested with CMs, almost all were tested with nonionic monomers $(\mathrm{n}=23)$, except for 2 patients (No. 12 and 24, table 4) who were tested with the nonionic dimer iodixanol. Further skin tests with alternative CMs were offered to 4 patients who were positive to the culprit CM. Three of them did not consent to further skin tests with alternative CMs. Only 1 patient (No. 12, table 4) was tested with an alternative CM, which gave a positive result, and she did not consent to further skin testing with another CM.

The control group consisted of 37 age- and gendermatched individuals (26 females and 11 males; mean age $51 \pm 11.35$ years), of whom 19 had never been exposed to a CM and 18 had tolerated radiological evaluation with $\mathrm{CM}$ application. Of the 37 controls, skin testing was performed with iohexol in 27 subjects (73\%), iopromide in 5 subjects (13.5\%), iomeprol in 3 subjects (8.1\%) and iodixanol in 2 subjects (5.4\%). SPTs and IDTs were positive in 2 of the 18 previously exposed controls and 3 of the 19 unexposed controls.

When we excluded the patients who were premedicated and tested with an alternative CM, there remained 10 patients with immediate reactions and 7 patients with nonimmediate reactions (No. 8-24, table 4). None of the 10 patients with CM-induced immediate reactions (No. 8-11 and 16-21, table 4) demonstrated SPT positivity to the culprit CM. Early readings of IDTs were $2+$ positive in 2 cases (20\%; patients No. 16 and 19, table 4). However, SPT positivity with a CM was observed in 2 individuals (5.4\%) who were both unexposed controls, and early readings of IDTs were also positive in 3 individuals (8.6\%) who were all exposed controls.

Accordingly, the sensitivity of the SPT was $0 \%$, specificity was $94.6 \%$ (95\% CI $80.5-99.1 \%$ ), PPV was $0 \%$ and NPV was $77.8 \%$ (95\% CI 62.5-88.3\%) for the diagnosis of immediate reaction to a $\mathrm{CM}$. For the early readings of IDTs, sensitivity was $20 \%$ (95\% CI 3.5-55.8\%), specificity was $91.4 \%$ (95\% CI 75.8-97.8\%), PPV was $40 \%$ (95\% CI 7.3-82.9\%) and NPV was 80\% (95\% CI 63.9-90.4\%) (fig. 1).

Positivity with the culprit agent (diluted 1:10) was observed on the delayed reading of the IDT in only 1 (14.3\%; patient No. 12, table 4 ) of the 7 nonpremedicated patients 
Table 4. Details of skin test results in patients who reacted to the CM used

\begin{tabular}{|c|c|c|c|c|c|c|c|c|c|c|c|c|c|c|c|}
\hline \multirow{2}{*}{$\begin{array}{l}\mathrm{Pa}- \\
\text { tient } \\
\text { No. }\end{array}$} & \multirow{2}{*}{$\begin{array}{l}\text { Age } \\
\text { years }\end{array}$} & \multirow{2}{*}{$\begin{array}{l}\text { Gen- } \\
\text { der }\end{array}$} & \multirow{2}{*}{ Reaction type } & \multirow{2}{*}{$\begin{array}{l}\text { Severity } \\
\text { degree }\end{array}$} & \multirow{2}{*}{$\begin{array}{l}\text { Time } \\
\text { pe- } \\
\text { riod }^{1}\end{array}$} & \multirow{2}{*}{$\begin{array}{l}\text { Previous } \\
\text { CM ex- } \\
\text { posure }\end{array}$} & \multirow{2}{*}{ Comorbid diseases } & \multirow{2}{*}{$\begin{array}{l}\text { Drug } \\
\text { allergy }\end{array}$} & \multirow{2}{*}{$\begin{array}{l}\text { Ato- } \\
\text { py }\end{array}$} & \multirow{2}{*}{$\begin{array}{l}\text { ESS } \\
\text { patch }\end{array}$} & \multirow[t]{2}{*}{ Tested CM } & \multicolumn{4}{|c|}{ CM skin testing } \\
\hline & & & & & & & & & & & & SPT & $\begin{array}{l}\mathrm{IDT}^{2} \\
(15 \mathrm{~min})\end{array}$ & $\begin{array}{l}\text { IDT }^{3} \\
(72 \mathrm{~h})\end{array}$ & PT \\
\hline 1 & 36 & $\mathrm{~F}$ & immediate & grade 1 & $7 y$ & - & asthma, allergic rhinitis & - & + & nickel + & ioxilan $^{4}$ & - & - & - & $\mathrm{ND}$ \\
\hline 2 & 63 & $\mathrm{M}$ & immediate & grade 1 & $37 y$ & + & hypertension, venom allergy & $y+$ & + & - & ioversal $^{4}$ & - & - & - & $\mathrm{ND}$ \\
\hline 3 & 63 & $\mathrm{~F}$ & immediate & grade 2 & $22 \mathrm{y}$ & + & thyroid disease & - & - & - & iohexol $^{4}$ & - & - & - & $\mathrm{ND}$ \\
\hline 4 & 41 & $\mathrm{~F}$ & immediate & grade 2 & $2 y$ & + & - & - & + & ND & iohexol $^{4}$ & - & - & - & ND \\
\hline 5 & 54 & $\mathrm{~F}$ & nonimmediate & severe & $1 \mathrm{y}$ & - & $\begin{array}{l}\text { hypertension, coronary } \\
\text { artery disease, diabetes } \\
\text { mellitus }\end{array}$ & - & - & - & iohexol $^{4}$ & - & - & - & - \\
\hline 6 & 52 & $\mathrm{~F}$ & nonimmediate & moderate & $4 y$ & - & psychiatric disease, asthma & - & - & - & iohexol $^{5}$ & - & - & - & - \\
\hline 7 & 69 & $\mathrm{~F}$ & nonimmediate & moderate & $4 y$ & + & $\begin{array}{l}\text { psychiatric disease, } \\
\text { hypertension, coronary } \\
\text { artery disease }\end{array}$ & - & - & - & iohexol $^{5}$ & - & - & - & - \\
\hline 8 & 51 & $\mathrm{M}$ & immediate & grade 1 & $2 \mathrm{~m}$ & + & urticaria & - & - & ND & iohexol $^{5}$ & - & - & & $\mathrm{ND}$ \\
\hline 9 & 45 & $\mathrm{~F}$ & immediate & grade 2 & $3 \mathrm{~m}$ & - & contact dermatitis, asthma & - & + & ND & iohexol $^{5}$ & - & - & - & $\mathrm{ND}$ \\
\hline 10 & 65 & $\mathrm{~F}$ & immediate & grade 1 & $2 \mathrm{~m}$ & - & hypertension & - & - & ND & iohexol $^{5}$ & - & - & - & ND \\
\hline 11 & 66 & $\mathrm{~F}$ & immediate & grade 1 & $2 \mathrm{~m}$ & + & hypertension & - & - & ND & iohexol $^{5}$ & - & - & - & $\mathrm{ND}$ \\
\hline 12 & 55 & $\mathrm{~F}$ & nonimmediate & moderate & $3 \mathrm{~m}$ & + & hypertension & - & - & - & $\begin{array}{l}\text { 1. } \text { iohexol }^{5} \\
\text { 2. iodixanol }\end{array}$ & ${ }_{-}^{-}$ & $\begin{array}{l}- \\
-\end{array}$ & $\begin{array}{l}+1: 10 \\
+1: 10\end{array}$ & - \\
\hline 13 & 58 & $\mathrm{~F}$ & nonimmediate & mild & $2 \mathrm{~m}$ & + & $\begin{array}{l}\text { hypertension, coronary } \\
\text { artery disease, thyroid } \\
\text { disease }\end{array}$ & - & NC & $\mathrm{NC}$ & iopromide $^{5}$ & - & - & - & $\mathrm{NC}$ \\
\hline 14 & 34 & $\mathrm{M}$ & nonimmediate & moderate & $2 \mathrm{~m}$ & - & - & - & - & $\mathrm{NC}$ & iohexol $^{5}$ & - & - & - & $\mathrm{NC}$ \\
\hline$\overline{15}$ & 18 & $\mathrm{~F}$ & nonimmediate & moderate & $2 \mathrm{~m}$ & + & - & - & - & $\mathrm{NC}$ & iohexol $^{5}$ & - & - & - & NC \\
\hline 16 & 55 & $\mathrm{M}$ & immediate & grade 2 & $2 \mathrm{~m}$ & - & coronary artery disease & - & - & ND & iopromide $^{5}$ & - & $+1: 10$ & & $\mathrm{ND}$ \\
\hline 17 & 40 & M & immediate & grade 2 & $2 \mathrm{~m}$ & - & allergic rhinitis & - & + & ND & iohexol $^{5}$ & - & - & - & ND \\
\hline 18 & 69 & $\mathrm{~F}$ & immediate & grade 1 & $2 \mathrm{~m}$ & - & asthma & - & - & ND & iopromide $^{5}$ & - & - & - & $\mathrm{ND}$ \\
\hline 19 & 42 & $\mathrm{~F}$ & immediate & grade 1 & $3 \mathrm{~m}$ & - & asthma & - & + & ND & iohexol $^{5}$ & - & $+1: 100$ & & $\mathrm{ND}$ \\
\hline 20 & 25 & $\mathrm{~F}$ & immediate & grade 2 & $3 \mathrm{~m}$ & - & asthma & - & - & ND & iohexol $^{5}$ & - & - & - & ND \\
\hline 21 & 63 & $\mathrm{M}$ & immediate & grade 2 & $2 \mathrm{~m}$ & + & $\begin{array}{l}\text { hypertension, coronary } \\
\text { artery disease }\end{array}$ & - & - & ND & iohexol $^{5}$ & - & - & - & $\mathrm{ND}$ \\
\hline 22 & 75 & $\mathrm{~F}$ & nonimmediate & moderate & $2 \mathrm{~m}$ & + & $\begin{array}{l}\text { hypertension, thyroid } \\
\text { disease, gastrointestinal } \\
\text { disease }\end{array}$ & - & - & - & iomeron $^{5}$ & - & - & - & $+1: 1,000$ \\
\hline 23 & 33 & $\mathrm{~F}$ & nonimmediate & mild & $2 \mathrm{~m}$ & + & thyroid disease & - & - & - & iohexol $^{5}$ & - & - & - & - \\
\hline 24 & 52 & $\mathrm{~F}$ & nonimmediate & mild & $2 \mathrm{~m}$ & - & hypertension & - & - & - & iodixanol $^{5}$ & - & - & - & - \\
\hline
\end{tabular}

For positive skin test reactions, the dilution of the CM that elicited the positive response is also shown. ESS = European standard series; $\mathrm{y}=\mathrm{years} ; \mathrm{m}=$ months; $\mathrm{NC}=$ no consent; $\mathrm{ND}=$ not done.

${ }^{1}$ The time period between the reaction and testing. ${ }^{2}$ Early readings of IDTs. ${ }^{3}$ Delayed readings of IDTs. ${ }^{4}$ Alternative CM. ${ }^{5}$ Culprit CM.

with nonimmediate reactions (No. $12-15$ and $22-24$, table 4). It was not positive in any of the control subjects. PT positivity was observed in only 1 patient (patient No. 22 , table 4 ). She had a moderate nonimmediate reaction with angioedema of the eyelids, generalized itching and maculopapular lesions $24 \mathrm{~h}$ after iomeron administration and was positive with all concentrations of iomeron tested on the PT. There was no positivity in any of the controls.
Accordingly, for the diagnosis of nonimmediate reaction to a CM, the sensitivity of delayed readings of the IDTs with the culprit agent was $14.3 \%$ (95\% CI 0.8-57.9\%), specificity was $100 \%$ (95\% CI $87.7-100 \%$ ), PPV was $100 \%$ (95\% CI 5.5-100\%) and NPV was $85.4 \%$ (95\% CI 70.193.9\%). For the PT carried out with the culprit agent, sensitivity was $25 \%$ (95\% CI $1.3-78.1 \%$ ), specificity was $100 \%$ (95\% CI $73.2-100 \%)$, PPV was $100 \%$ (95\% CI $5.5-100 \%)$ and NPV was $82.4 \%$ (95\% CI 55.8-95.3\%) (fig. 1). 
Fig. 1. Usefulness of diagnostic tests performed in the study. Positive tests on SPTs $(\mathrm{n}=54)$ : controls $2 / 37$; immediate reactions $0 / 10$; nonimmediate reactions $0 / 7$. Positive tests on early readings of IDTs $(\mathrm{n}=52)$ : controls $3 / 35$; immediate reactions 2/10; nonimmediate reactions $0 / 7$. Positive tests on delayed readings of IDTs $(\mathrm{n}=52)$ : controls $0 / 35$; immediate reactions $0 / 10$; nonimmediate reactions $1 / 7$. Positive tests on PTs $(\mathrm{n}=18)$ : controls $0 / 14$; nonimmediate reactions $1 / 4$.

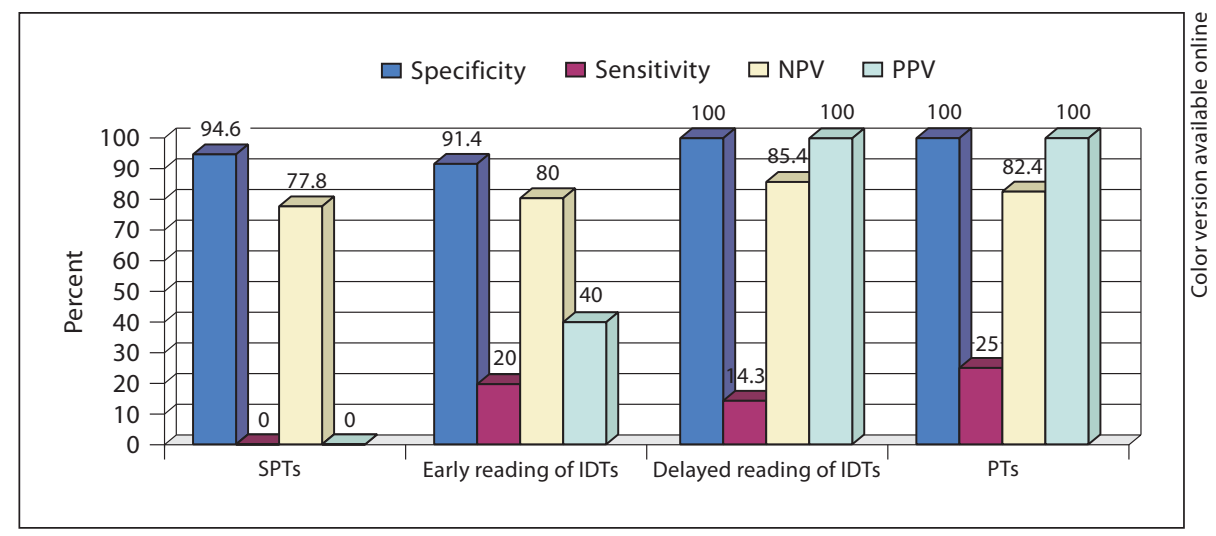

\section{Discussion}

In this study, the overall prevalence of hypersensitivity reactions to CMs, including past reactions, was 3.62\%, and the current prevalence of hypersensitivity reactions to nonionic CMs was $0.72 \%$. The introduction of nonionic $\mathrm{CMs}$ has been associated with reduced adverse reactions to CM administration [4, 6, 7-9]. The overall rate of adverse reactions to iopromide, a nonionic monomer, was reported to be $1.5 \%$ in a large-scale surveillance study [4]. Dahlstrom et al. [28] reported the rate of adverse reactions to another nonionic monomer, iohexol, to be $0.8 \%$. As nonionic CMs, iohexol, iomeprol and iopromide were the most common CMs in our study, and our current prevalence rate was comparable with the data reported by studies with nonionic CMs [4, 6-9]. However, the past prevalence rate was relatively higher than current reactions to CMs during the 1-year study period (2.92 vs. $0.72 \%)$. This may be related to recall bias or the type of CMs used at that time. Only 4 of the previous CM reactors recalled the culprit CMs, which were ionic and nonionic, and no data were available about the relevant $\mathrm{CM}$ for the other previous reactors.

CM-related severe immediate reactions are extremely rare compared to mild reactions $[4,6,21]$. Studies have shown that the rate of severe and mild immediate reactions to high-osmolar ionic CMs ranges from 0.04 to $0.05 \%$ and from 3.8 to $12.7 \%$, respectively $[6,7,14]$. Similarly, a clear variability ranging from 0.5 to $23 \%$ for the frequency of nonimmediate reactions has been reported $[7,22]$. Heterogeneity with regard to classifications and study design makes it difficult to make comparisons between these studies and highlights the risk of false positivity. Our prevalence rates for immediate and nonimmediate reactions to CMs were in agreement with these data.
Several risk factors associated with hypersensitivity reactions to CMs have been reported. These are both patient related, such as age, gender, atopy and accompanying atopic or nonatopic diseases, and drug related, such as dose, group or route of administration [4, 6-10, 23]. As with findings from these studies, female gender resulted in a 2-fold increase in the prevalence of CM hypersensitivity in our study. Other significant risk factors were doctor-diagnosed asthma and drug and food allergy. Contrary to the common false belief about the connection between iodine and seafood allergy, there was no specific food that was associated with hypersensitivity to CMs in our study, but the number of patients with a history of food allergy was very limited [24, 25]. The frequency of associated drug allergy with CM hypersensitivity is well known $[8,10,13,26]$, as we have observed in our study. Doctor-diagnosed psychiatric disorders constituted another prominent risk factor in our study. This has not been addressed in the same way in any previous studies, but anxiety was reported as a risk factor for CM hypersensitivity reaction in one old study [27]. Postmarketing studies reported a significant difference between intraarterial and intravenous administration of CMs, with higher rates of reactions associated with intraarterial application $[4,5,28]$. It is difficult for us to comment on that issue, because in our study, the majority of CMs were administered by the intravenous route.

CM-related immediate reactions constitute about 70\% of cases characterized, from erythema, urticaria and angioedema to anaphylaxis [14]. Although a number of clinical presentations have been defined as nonimmediate reactions, the majority are unrelated with the CM, except skin reactions [22]. The skin represents the site most affected by immediate/nonimmediate reactions, among which pruritus, mild urticaria and maculopapular rashes 
are the most frequently reported $[14,20]$. In our CM reactors, urticarial rush, erythema and angioedema were common in both types of reactions, while maculopapular rashes were specific for nonimmediate reactions.

Some recent data indicated the role of immune mechanisms in CM-induced immediate/nonimmediate reactions. Specific IgE measurement, skin tests and lymphocyte and basophil activation tests have been performed to define the underlying immune mechanism $[2,12,13,19$, $23,29,30]$. With respect to skin tests with CMs, SPTs and early readings of IDTs have been used in the diagnosis of immediate-type reactions $[2,31]$. Positive reactions to SPTs are rare, but sensitivity to IDTs of up to $25 \%$ has been reported [31]. Similarly, in our study SPTs were not positive in any patients with an immediate reaction, but early readings of IDTs had a sensitivity of $20 \%$, which was relatively lower than that demonstrated by Brockow et al. [31]. Although our positivity criteria for SPTs were similar but different to those for IDTs in that recent study [31], the 2 patients with $2+$ reactions on early readings of IDTs also fit the positivity criteria of the current study [31]. Positive reactions on SPTs and IDTs have been associated with severe systemic reactions in some cases, although not in others $[2,13,23,31]$. Our patients with immediate reactions had grade 1 and 2 severities, which might have contributed to the low level of sensitivity. Specificities of IDTs for immediate reactions have ranged from 96.3 to $97.6 \%[31,32]$. Our specificity rates were quite close to previously reported rates. With regard to the PPV and NPV, both tests had a high probability of false positivity but a moderate probability of false negativity.

Previous studies have demonstrated that up to $60 \%$ of patients with nonimmediate skin reactions to CMs can be diagnosed by delayed readings of IDTs and PTs [14, 31]. Additionally, skin tests are more frequently positive in patients with maculopapular eruption than in patients with nonimmediate urticarial lesions [31,33]. The most common skin reaction in our patients with nonimmediate reactions was maculopapular exanthems, followed by urticarial-like skin rashes and angioedema. The sensitivities of delayed IDTs and PTs were lower than those of a recent multicenter study [31], but specificity was in accordance with that study. Considering the perfect PPV and relatively weak NPV, both tests had a probability of false negativity.

The role of the time interval between the reaction and testing has been reported previously $[2,31]$. In a recent multicenter study, up to $50 \%$ of immediate reactors and up to $47 \%$ of nonimmediate reactors had positive skin tests if they were tested within 2-6 months of the reac- tion [31]. Further, higher positivity rates on skin tests have been obtained with the culprit drug [31]. In line with this observation regarding the time interval between the reaction and skin testing, none of the patients in our study with a history of a reaction to a CM more than 6 months previously was positive on skin tests, and skin test positivity to CMs was observed only among patients who were tested between 2 and 3 months after the reaction and among patients who were tested with culprit agents.

The effectiveness of premedication for prevention of CM-induced reactions is under discussion $[4,5,8,34]$. Since the NPV is unknown, it remains unclear whether a skin test-negative CM can be safely administered [31]. Although we premedicated 6 previous reactors, the limited number of patients in our study prevents us from drawing any conclusion on the preventive effect of premedication.

Our study has both disadvantages and advantages. The first disadvantage was the retrospective data collection on previous reactions to CMs. We had to rely on the patients' statements; therefore, there might be a high likelihood of recall bias. Secondly, the sample size was quite small compared to large-scale, postmarketing studies. However, they were all multicenter or international studies. Our national, single-center study gave us a fairly good idea regarding the incidence of CM-induced hypersensitivity reactions for the first time in our country. On the other hand, studies investigating the usefulness of skin tests in patients with CM-related allergy-like reactions are mostly limited to case reports and there are few well-documented studies [2, 16, 19, 31]. We believe that our study deserves attention since it has quite a large number of $\mathrm{CM}$ reactors who were all skin tested with mostly culprit CMs. All these studies, including our own experience, have used a history of hypersensitivity reaction to a $\mathrm{CM}$ as a gold standard and calculated sensitivity as the percentage of positive skin tests among these patients. However, it is difficult to be certain of skin test sensitivity without a confirmative provocation test. One advantage of our study is that we had subjects who had been exposed to a CM without reacting and healthy subjects who had never been exposed to a CM as controls. To the best of our knowledge, only a recent European Network of Drug Allergy/European Academy of Allergy and Clinical Immunology study used such subjects as controls who contributed to establishing the value of skin tests. We also provide data, for the first time, about the PPV and NPV of skin tests, which are necessary to consider false negativity and positivity of the results. 
In conclusion, our findings are comparable with the data reported previously regarding the incidence and profile of CM hypersensitivity and risk factors associated with it. Skin testing with CMs has a high specificity, but its role in diagnosis is limited by a low sensitivity in mild to moderate reactions to CMs.

\section{Acknowledgements}

We would like to thank Asuman Guzelant, MD, for her assistance in statistical analyses and nurses Ozlem Giris and Serap Tuncer for their assistance in skin test administration. This study was supported by the Ankara University Commission of Scientific Research Project (2006-08-09-027HPD).

\section{References}

-1 Christiansen C: X-ray contrast media - an overview. Toxicology 2005;209:185-187.

$\checkmark 2$ Kvedarine V, Martins P, Rouanet L, Demoly P: Diagnosis of iodinated contrast media hypersensitivity: results of a 6-year period. Clin Exp Allergy 2006;36:1072-1077.

$\checkmark 3$ Bettmann MA: Frequently asked questions: iodinated contrast agents. Radiographics 2004;24(suppl 1):3-10.

$\checkmark 4$ Kopp AF, Mortele KJ, Cho YD, Palkowitsch P, Bettmann MA, Claussen CD: Prevalence of acute reactions to iopromide: postmarketing surveillance study of 74,717 patients. Acta Radiol 2008;49:902-911.

5 Bettmann MA, Heeren T, Greenfield A, Goudey C: Adverse events with radiographic contrast agents: results of the SCVIR Contrast Agent Registry. Radiology 1997;203:611-620.

6 Katayama H, Yamaguchi K, Kozuka T, Takashima T, Seez P, Matsuura K: Adverse reactions to ionic and nonionic contrast media. A report from the Japanese Committee on the Safety of Contrast Media. Radiology 1990; 175:621-628.

7 Idèe J-M, Pines E, Prigent P, Corot C: Allergy-like reactions to iodinated contrast agents. A critical analysis. Fundam Clin Pharmacol 2005;19:263-281.

$\checkmark 8$ Dawson P: Adverse reactions to intravascular contrast agents. BMJ 2006;333:663-664.

19 Wolf GL, Arenson RL, Cross AP: A prospective trial of ionic vs nonionic contrast agents in routine clinical practice: comparison of adverse effects. AJR Am J Roentgenol 1989; 152:939-944.

$>10$ Hagan JB: Anaphylactoid and adverse reactions to radiocontrast agent. Immunol Allergy Clin North Am 2003;24:507-519.

-11 Guéant-Rodriguez RM, Romano A, Barbaud A, Brockow K, Guéant JL: Hypersensitivity reactions to iodinated contrast media. Curr Pharm Des 2006;12:3359-3372.

$\checkmark 12$ Torres MJ, Mayorga C, Cornejo-Garcia JA, Lopez S, Chaves P, Rondon C, Fernandez T, Blanca M: Monitoring non-immediate allergic reactions to iodine contrast media. Clin Exp Allergy 2008;152:233-238.

-13 Kanny G, Pichler W, Morisset M, Franck P, Marie B, Kohler C, Renaudin JM, Beaudouin E, Laudy JS, Moneret-Vautrin DA: T cell-mediated reactions to iodinated contrast media: evaluation by skin and lymphocyte activation tests. J Allergy Clin Immunol 2005;115: 179-185.
14 Brockow K, Christiansen C, Kanny G, Clément O, Barbaud A, Bircher A, Dewachter P, Guéant JL, Rodriguez Guéant RM, MoutonFaivre C, Ring J, Romano A, Sainte-Laudy J, Demoly P, Pichler WJ; ENDA; EAACI interest group on drug hypersensitivity: management of hypersensitivity reactions to iodinated contrast media. Allergy 2005;60:150 158.

15 Vernassiere C, Trechot P, Commun N, Schmutz JL, Barbaud A: Low negative predictive value of skin tests in investigating delayed reactions to radio-contrast media. Contact Dermatitis 2004;50:359-366.

16 Demoly P, Kropf R, Bircher A, Pichler WJ: Drug hypersensitivity: questionnaire. Allergy 1999;54:999-1003.

17 Ring J, Messmer K: Incidence and severity of anaphylactoid reactions to colloid volume substitutes. Lancet 1977;i:466-469.

18 Barbaud A, Goncalo M, Bruynzeel D, Bircher A: Guidelines for performing skin tests with drugs in the investigation of cutaneous adverse drug reactions. Contact Dermatitis 2001;45:321-328.

19 Trcka J, Schmidt C, Seitz CS, Bröcker EB, Gross GE, Trautmann A: Anaphylaxis to iodinated contrast material: nonallergic hypersensitivity or IgE-mediated allergy? AJR Am J Roentgenol 2008;190:666-670.

20 Brockow K: Contrast media hypersensitivity - scope of the problem. Toxicology 2005; 209:189-192.

21 Caro JJ, Trindade E, McGregor M: The risks of death and of severe nonfatal reactions with high- versus low-osmolality contrast media: a meta-analysis. AJR Am J Roentgenol 1991; 156:825-832.

22 Webb JA, Stacul F, Thomsen HS, Morcos SK; Members Of The Contrast Media Safety Committee Of The European Society Of Urogenital Radiology: late adverse reactions to intravascular iodinated contrast media. Eur Radiol 2003;13:181-184.

23 Moneret-Vautrin DA, Kanny G, Morisset M, Beaudouin E, Renaudin JM: Anaphylactoid reactions and late skin reactions to iodinated contrast media: present state of the question - idea development (in French). Rev Med Interne 2001;22:969-977.
24 Sicherer SH: Risk of severe allergic reactions from the use of potassium iodide for radiation emergencies. J Allergy Clin Immunol 2004;114:1395-1397.

25 Canter LM: Anaphylactoid reactions to radiocontrast media. Allergy Asthma Proc 2005;26:199-203.

26 Asero R: Multiple drug allergy syndrome: a distinct clinical entity. Curr Allergy Rep 2001;1:18-22.

27 Lalli AF: Urographic contrast media reactions and anxiety. Radiology 1974;112:267271.

$\checkmark 28$ Dahlstrom K, Shaw DD, Clauss W, Andrew E, Sveen K: Summary of U.S. and European intravascular experience with iohexol based on the clinical trial program. Invest Radiol 1985;20(1 suppl):S117-S121.

-29 Laroche D, Aimone-Gastin I, Dubois F, Huet H, Gérard P, Vergnaud MC, Mouton-Faivre $\mathrm{C}$, Guéant JL, Laxenaire MC, Bricard $\mathrm{H}$ : Mechanisms of severe, immediate reactions to iodinated contrast material. Radiology 1998;209:183-190.

30 Mita H, Tadokoro K, Akiyama K: Detection of $\mathrm{IgE}$ antibody to a radiocontrast medium. Allergy 1998;53:1133-1140.

- 31 Brockow K, Romano A, Aberer W, Bircher AJ, Barbaud A, Bonadonna P, Faria E, Kanny G, Lerch M, Pichler WJ, Ring J, Rodrigues Cernadas J, Tomaz E, Demoly P, Christiansen C; European Network of Drug Allergy and the EAACI interest group on drug hypersensitivity: skin testing in patients with hypersensitivity reactions to iodinated contrast media - a European multicenter study. Allergy 2009;64:234-241.

>32 Guillén Toledo J, Guido Bayardo R: Anamnesis and skin test to prevent fatal reactions to iodinated contrast media (in Spanish). Rev Alerg Mex 2000 47:22-25.

33 Lerch M, Keller M, Britschgi M, Kanny G, Tache V, Schmid DA, Beeler A, Gerber BO, Luethi M, Bircher AJ, Christiansen C, Pichler WJ: Cross-reactivity patterns of T cells specific for iodinated contrast media. J Allergy Clin Immunol 2007;119:15291536

34 Wolf GL, Mishkin MM, Roux SG, Halpern EF, Gottlieb J, Zimmerman J, Gillen J, Thellman C: Comparison of the rates of adverse drug reactions. Ionic contrast agents, ionic agents combined with steroids, and nonionic agents. Invest Radiol 1991;26:404-410. 